MKG-Chirurg 2008 · 1:173-173

DOI 10.1007/s12285-008-0039-0

Online publiziert: 25 . Oktober 2008

(c) Springer Medizin Verlag 2008

\author{
E. Esser ${ }^{1} \cdot$ F. Muggenthaler ${ }^{2}$ \\ ${ }^{1}$ ICOS ImplantatCentrum Osnabrück \\ ${ }^{2}$ Fontana-Klinik an den Thermen, Freiburg
}

\title{
Ästhetische Gesichtschirurgie
}

In dieser Ausgabe unserer neuen Zeitschrift „Der MKG-Chirurg“ finden Sie das Schwerpunktthema „ästhetische Gesichtschirurgie" mit Beiträgen profilierter $\mathrm{Au}$ toren, die über ihre persönlichen Erfahrungen berichten. Damit soll unser fachlicher Anspruch auf ästhetische Eingriffe im Gesichtsbereich betont werden, denn kein anderes Fachgebiet ist besser und spezieller ausgebildet oder verfügt über ein breiteres Spektrum. In der Vorbereitung des Heftes wurde deutlich, dass sich eine zunehmende Zahl von MKG-Chirurgen sehr erfolgreich der ästhetischen Gesichtschirurgie zugewendet hat. Die zertifizierte Fortbildung wird diese Entwicklung und die öffentliche Wahrnehmung der MKG-Chirurgie weiter fördern. Die im Jahr 2006 mit 25 Teilnehmern begonnene erste Kursreihe wurde zwischenzeitlich mit der Verleihung der Zertifikate abgeschlossen. Der zweite Kurs hat im Dezember 2007 mit 30 Teilnehmern begonnen. Die Akademie plant aufgrund der großen Nachfrage weitere Kurse. Damit ist ein entscheidender Schritt zur Qualitätssicherung und zur Dokumentation unserer Fachkompetenz getan.

\section{$>$ Ästhetische Gesichtschirurgie: die wissenschaftliche Herausforderung unseres Fachgebietes}

Elektive Operationen unterliegen zwangsläufig besonders hohen medizinethischen Anforderungen, die sich allerdings auch bei einseitiger Auslegung als „Ethikfalle" erweisen können. Es ergibt sich daher die Notwendigkeit, die ästhetische Gesichtschirurgie auch als wissenschaftliche Herausforderung unseres Fachgebietes zu begreifen. Dazu sollten die Alterungsvorgänge in Art, Vektorrichtung und Dimension analysiert sowie chirurgische Korrekturverfahren und Unterfütterungskonzepte evaluiert werden.

Wenn wir die Grundsätze einer strukturierten Aus- und Weiterbildung und einer wissenschaftlichen Bearbeitung befolgen, wird die ästhetische Gesichtschirurgie in der Hand des MKG-Chirurgen als seriös wahrgenommen werden und eine hohe Anerkennung gewinnen.

\section{Korrespondenzadresse \\ Prof. Dr. Dr. E. Esser}

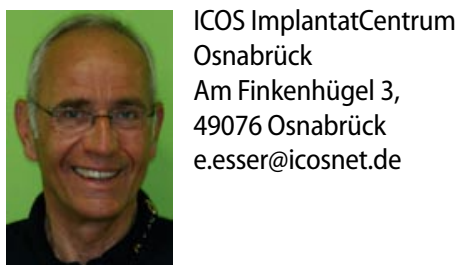

\section{Dr. Dr. F. Muggenthaler}

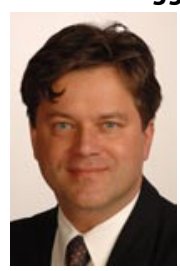

Fontana-Klinik an den Thermen An den Heilquellen 8, 79111 Freiburg office@muggenthaler.net 